\title{
Robust Filtering for A Class of Nonlinear Stochastic Systems with Probability Constraints
}

\author{
Lifeng Ma*, Zidong Wang, Hak-Keung Lam, Fuad E. Alsaadi and Xiaohui Liu
}

\begin{abstract}
This paper is concerned with the probability-constrained filtering problem for a class of time-varying nonlinear stochastic systems with estimation error variance constraint. The stochastic nonlinearity considered is quite general that is capable of describing several well-studied stochastic nonlinear systems. The second-order statistics of the noise sequence are unknown but belong to certain known convex set. The purpose of this paper is to design a filter guaranteeing a minimized upper-bound on the estimation error variance. The existence condition for the desired filter is established, in terms of the feasibility of a set of difference Riccati-like equations, which can be solved forward in time. Then, under the probability constraints, a minimax estimation problem is proposed for determining the suboptimal filter structure that minimizes the worst-case performance on the estimation error variance with respect to the uncertain second-order statistics. Finally, a numerical example is presented to show the effectiveness and applicability of the proposed method.
\end{abstract}

\section{Keywords}

Probability constraint, Time-varying systems, Measurements degradation, Stochastic nonlinearity, Parameter uncertainty

\section{INTRODUCTION}

For several decades, nonlinear stochastic systems have been attracting tremendous interest in the system science and control community due to their extensive applications in a variety of areas such as communication, transportation, manufacturing, building automation, computing, automotive, and chemical industry [1-3]. Nowadays, nonlinear stochastic systems are playing more and more prevalent roles in various branches of theoretical research and engineering applications, especially those related to signal processing and stochastic control [4-7]. As is well known, in many stochastic filtering problems such as the maneuvering target tracking problem, the performance requirements are naturally expressed as upper-bounds on the filtering error variances, see, e.g. [8-10]. Unfortunately, the traditional filtering design techniques (e.g. $\mathcal{H}_{\infty}$ filtering

This work was supported in part by the Royal Society of the U.K., the National Natural Science Foundation of China under Grants 61304010 and 61329301, the Natural Science Foundation of Jiangsu Province under Grant BK20130766, the Postdoctoral Science Foundation of China under Grant 2014M551598, International Postdoctoral Exchange Fellowship from the China Postdoctoral Council, and Alexander von Humboldt Foundation of Germany.

L. Ma is with the School of Automation, Nanjing Univerity of Science and Technology, Nanjing 210094, China. (Email: malifeng@njust.edu.cn)

Z. Wang is with the Department of Computer Science, Brunel University London, Uxbridge, Middlesex, UB8 3PH, United Kingdom. He is also with the Communication Systems and Networks (CSN) Research Group, Faculty of Engineering, King Abdulaziz University, Jeddah 21589, Saudi Arabia. (Email: Zidong.Wang@brunel.ac.uk)

H.-K. Lam is with Department of Informatics, School of Natural \& Mathematical Science, King's College London, Strand Campus, WC2R 2LS, United Kingdom.

F. E. Alsaadi is with the Communication Systems and Networks (CSN) Research Group, Faculty of Engineering, King Abdulaziz University, Jeddah 21589, Saudi Arabia.

X. Liu are with the Department of Computer Science, Brunel University London, Uxbridge, Middlesex, UB8 3PH, United Kingdom.

${ }^{*}$ Corresponding author. 
algorithm and minimum variance filtering approach) are no longer effective in handling variance-constrained filtering problem since they lack a convenient avenue for directly imposing design objectives stated in terms of the upper-bounds on the individual error variance values. The covariance control theory [11] developed in the late 80s has provided a direct filtering methodology for achieving the individual error variance constraints imposed on the states. It should be emphasized that, since then, due to the elegancy and convenience in dealing with variance-related problems, the covariance control theory has been serving as a practical method for variance-constrained control/filtering design as well as a foundation for linear system theory.

In recent years, there has been a renewed research interest on the design technique for the varianceconstrained filtering problem for more complicated systems such as nonlinear stochastic systems and timevarying stochastic systems $[14,15]$. However, it should be pointed out that limited work has been reported due mainly to the fact that either nonlinearities or time-varying parameters exhibit much more complicated dynamics than that resulting from the traditional linear time-invariant systems, and this has inevitably led to unanticipated difficulties in handling the state/output/error covariance. In [12], the error variance-constrained filtering problem has been solved for linear time-varying stochastic systems in terms of certain Riccati equations. For a special type of nonlinear stochastic systems, a sufficient condition has been proposed in [13] to the existence of an optimal filter expressed by the feasibility of a set of Riccati-like equations, and the optimal filter parameters can be obtained by the gradient method. Unfortunately, filtering problems for more complex nonlinear time-varying systems with variance constraints have not yet been investigated adequately.

On another research frontier, the past several decades have witnessed the extensive studies and applications of the celebrated Kalman filtering in the area of signal processing, see [16-19] and the references therein. As is well known, the standard Kalman filtering algorithm is only applicable to the systems with precise system models and known statistics of the noises, and this has placed certain restriction in practical engineering. To improve the robustness of the filter performances, in recent years, much research effort has been devoted to the robust filtering problem in the branches of stochastic estimation and control theory [20-24]. Several techniques have been proposed in the literature, among which the well-known minimax estimation approach has stirred special research interests due to its robustness against the system uncertainties. Such an algorithm aims to find an optimal scheme such that the worst-case performance over all possible values of the uncertain parameters is minimized, see $[25,26]$ and the references therein.

It is worth mentioning that, though the minimax algorithm appears to be elegant, it suffers from certain limitations. The most notable limitation is arguably the conservatism since the minimization is implemented subject to the worst-case situation that is very likely to be a small probability event. As a result, much work has been done to reduce the conservatism and several approaches have been exploited. A particular method incorporating the known constraints (imposed on the system states) into the minimax estimation framework has proven to be fairly effective. For example, in [27,28], a receding horizon approximation method has been proposed to give an optimal filtering algorithm while taking the known constraints on system state into account, thereby largely reducing the conservatism. Recently, the probability constraint imposed on the system measured output has been incorporated in [29] to design a minimax filtering algorithm guaranteeing a minimal worst-case performance index with respect to the unknown disturbance.

It is worth mentioning that the discussion on the system uncertainties has mainly concerned with the unknown external disturbance (e.g. white Gaussian noises with unknown covariances mentioned above in [29] or random factors with unknown covariances [30]) and other frequently occurred incomplete measurements have not been taken into adequate consideration. In practical systems within networked environments (e.g., 
sensor networks and networked control systems), due to various reasons such as sensor temporal failure, limited capacity of device or network transmission delay/loss, the measurement signals may have different networkinduced issues such as information loss, equipment failures and nonlinear disturbances. Such a phenomenon is often referred to as the measurement degradation, which would drastically deteriorate the system performance and therefore has attracted considerable research attention in the past few years, see [31-35] for some latest publications. Unfortunately, so far, to the best of our knowledge, the robust filtering problem for nonlinear stochastic systems with probability constraints on system outputs has not yet been thoroughly investigated, which still remains as a challenging problem.

In this paper, it is our objective to design a filter that achieves the prespecified variance constraints on the filtering errors over a finite horizon subject to a probability constraint imposed on the system output. The main contribution of this paper lies in the following two aspects. 1) A suboptimal filtering algorithm is proposed by taking the parameter uncertainty, the measurements degradation, the stochastic nonlinear disturbance and the noise with unknown covariance into simultaneous consideration. 2) The probability constraint imposed on system measured output is considered to reduce the conservatism of the proposed robust filtering algorithm. The rest of this paper is organized as follows: Section II formulates the suboptimal robust filter design problem. Section III presents a filter design algorithm such that the upper-bound of estimation error variance can be guaranteed. Section IV gives the solution to the addressed suboptimal robust filter design problem with probability constraint. Section V presents a numerical example. Section VI is our conclusion.

Natation The notation used here is fairly standard except where otherwise stated. $\mathbb{R}^{n}$ denotes the $n$ dimensional Euclidean space. The notation $X \geq Y$ (respectively $X>Y$ ) where $X$ and $Y$ are symmetric matrices, means that $X-Y$ is positive semi-definite (respectively positive definite). $\mathbb{E}\{x\}$ stands for the expectation of stochastic variable $x$ and $\mathbb{E}\{x \mid y\}$ for the expectation of $x$ conditional on $y$. The superscript " $\mathrm{T}$ " denotes the transpose. $\|a\|_{2}^{2}$ where $a$ is a vector represents $a^{\mathrm{T}} a$, while $\|a\|_{A}^{2}$ means $a^{\mathrm{T}} A a$. $\operatorname{tr}[A]$ means the trace of matrix $A$. $\operatorname{diag}\left\{F_{1}, F_{2}, \ldots, F_{n}\right\}$ denotes a block diagonal matrix whose diagonal blocks are given by $F_{1}, F_{2}, \ldots, F_{n}$.

\section{Problem Formulation}

Consider the following time-varying nonlinear stochastic system defined on $k \in[0, N]$ :

$$
\left\{\begin{aligned}
x_{k+1} & =A_{k} x_{k}+B_{k} \omega_{k}, \\
y_{k} & =\Theta_{k}\left(C_{k}+\Delta C_{k}\right) x_{k}+G_{k} g\left(x_{k}, k\right)+D_{k} \omega_{k}, \\
z_{k} & =L_{k} x_{k}+M_{k} \omega_{k}
\end{aligned}\right.
$$

where $x_{k} \in \mathbb{R}^{n}$ is the system state, $y_{k} \in \mathbb{R}^{m}$ and $z_{k} \in \mathbb{R}^{m}$ represent the measured outputs. $\omega_{k} \in \mathbb{R}^{r}$ represents the external disturbance which is a white Gaussian sequence with zero mean. The covariance of $\omega_{k}$ is denoted by $W_{k}$ which is unknown but belongs to a compact and convex set $\mathcal{W} . A_{k}, B_{k}, C_{k}, D_{k}, G_{k}, L_{k}$, and $M_{k}$ are known real time-varying matrices with appropriate dimensions. It is assumed that the mean and covariance of initial state $x_{0}$ are also known, characterized by $\bar{x}_{0}$ and $X_{0}$ respectively. Without loss of generality, it is assumed that $B_{k} W_{k} D_{k}^{\mathrm{T}}=0$.

The matrix $\Delta C_{k}$ represents the parameter uncertainty and satisfies:

$$
\Delta C_{k}=H_{k} F_{k} E_{k}, \quad F_{k}^{\mathrm{T}} F_{k} \leq I,
$$

where $H_{k}$ and $E_{k}$ are known matrices. The parameter uncertainty $\Delta C_{k}$ is said to be admissible if it satisfies $(2)$. 
The stochastic matrix $\Theta_{k}$ describes the phenomenon of multiple measurements degradation in the process of information retrieval from the sensor output. $\Theta_{k}$ is defined as

$$
\Theta_{k} \triangleq \operatorname{diag}\left\{\theta_{1 k}, \theta_{2 k}, \ldots, \theta_{m k}\right\}
$$

with $\theta_{j k}(j=1,2, \ldots, m)$ being $m$ mutually independent random variables which are also independent from $\omega(k)$. It is assumed that $\theta_{j k}$ has the probabilistic density function $p_{j}(s)$ on the interval $[0,1]$ with mathematical expectation $\bar{\theta}_{j k}$ and variance $\sigma_{j k}^{2}$.

Remark 1: In the system measurement process with multiple sensors, the stochastic matrix $\Theta_{k}$ describes the working status of these sensors. Notice that $\theta_{j k}$ has the probabilistic density function $p_{j}(s)$ on the interval $[0,1]$. In this case, the measurement output model in this paper is more general than those in existing literature where a Bernoulli distributed stochastic sequence is assumed to take values on 0 or 1 only. In our measurement model, when $\theta_{j k}=1$, it means that the $j$ th sensor is in good condition, otherwise there might be partial or complete sensor failure. More specifically, when $\theta_{j k}=0$, the sensor is totally out of order and the measurements are completely missing, while $0<\theta_{j k}<1$ means that we could only measure the output signals with reduced gains leading to degraded measurements. In this sense, the model (1) offers a comprehensive means to reflect systems complexity such as nonlinearities, stochasticity and data degraded from multiple sensors.

The nonlinear stochastic function $g\left(x_{k}, k\right)$ is assumed to satisfy:

$$
\begin{aligned}
\mathbb{E}\left\{g\left(x_{k}, k\right) \mid x_{k}\right\} & =0, \\
\mathbb{E}\left\{g^{\mathrm{T}}\left(x_{j}, j\right) g\left(x_{k}, k\right) \mid x_{k}\right\} & =0, \quad k \neq j \\
\mathbb{E}\left\{g^{\mathrm{T}}\left(x_{k}, k\right) g\left(x_{k}, k\right) \mid x_{k}\right\} & =\sum_{i=1}^{q} \Pi_{i k}\left(x_{k}^{\mathrm{T}} \Gamma_{i k} x_{k}\right)
\end{aligned}
$$

where $\Pi_{i k}$ and $\Gamma_{i k}(i=1,2, \ldots, q)$ are known semi-positive definite matrices with appropriate dimensions.

Remark 2: As discussed in [36], the nonlinear description (4) can cover several well-studied nonlinear stochastic systems, such as system with state-dependent multiplicative noises, nonlinear systems with random sequences whose powers depend on sector-bound nonlinear function of the state, nonlinear systems with a random sequence whose power depends on the sign of a nonlinear function of the state, to name just a few.

The measurement $z_{k}$ satisfies the following probability constraint:

$$
\operatorname{Prob}\left\{z_{k} \leq \varphi_{k}\right\} \geq \gamma_{k}
$$

where $\varphi_{k}$ and $\gamma_{k}$ are given vectors and the inequality holds in an element-wise manner.

It is noted that the system (1) has two different types of outputs, namely, $y_{k}$ and $z_{k}$. Here, $y_{k}$ is the usual system measurements that are subjected to certain imprecision resulting from variations of operating points, aging of devices, identification errors, abrupt changes of working circumstances and temporal failures, to name just a few. The measured output $y_{k}$ in the addressed model is comprehensive to accounts for parameter uncertainties, randomly occurring nonlinearities, external white noises as well as randomly occurring measurement degradations.

Remark 3: As discussed in [29], in this paper, $z_{k}$ is the output of interest that is assumed to satisfy the probability constraint (5). In practical engineering, based on our previous experience/knowledge, we might have high confidence that certain system output satisfies some upper bound constraints during the estimation interval. An adequate usage of such kind of additional knowledge, expressed in the form of (5), would definitely help improve the estimation performance. 
One practical application of the problem formulation (1)-(5) might be the target tracking problem. Nowadays, due to the high maneuverability of modern aircrafts, it is often the case that we will have to exploit as much information as possible from different sensing sources implemented distributively but connected via networks. In addition, sometimes we might receive the data shared by other allied tracking units. In such a setting, $y_{k}$ represents the physical data obtained from the sensing sources with adequate knowledge (i.e, the working condition, the device information, etc), which allows us to adopt a relatively accurate measurement model to describe the evolution precisely. On the other hand, $z_{k}$ stands for those information we are interested and we have a priori knowledge about it. In our case, we have certain confidence (quantified by probability) that $z_{k}$ is constrained by a known upper bound, and the probability constraint could be obtained and verified via experiments, training, or actual combat experiences.

Now, consider the following filter for the uncertain discrete time-varying nonlinear stochastic system (1):

$$
\mathscr{F}: \hat{x}_{k+1}=A_{f k} \hat{x}_{k}+K_{f k}\left(y_{k}-\bar{\Theta}_{k} C_{k} \hat{x}_{k}\right)
$$

where $\bar{\Theta}_{k} \triangleq \mathbb{E}\left\{\Theta_{k}\right\}$, and $\hat{x}_{k} \in \mathbb{R}^{n}$ is the state estimate, $A_{f k}$ and $K_{f k}$ are the filter parameters to be determined.

In this paper, it is our objective to design a finite-horizon filter of the structure (6), such that

(1) For all admissible parameter uncertainty, possible degraded measurements and nonlinear disturbances, there exists a sequence of positive-definite matrices $Q_{k}$ satisfying:

$$
\mathbb{E}\left\{\left(x_{k}-\hat{x}_{k}\right)\left(x_{k}-\hat{x}_{k}\right)^{\mathrm{T}}\right\} \leq Q_{k} .
$$

That is, at each time point $k$, the finite upper-bound on the state estimation error covariance is guaranteed. It is worth mentioning that, usually, the desired filter satisfying constraint (7) is not unique but belongs to certain set.

(2) The performance index defined by the following cost function

$$
J_{k}\left(\mathscr{F} ; W_{k}\right)=\operatorname{tr}\left[Q_{k+1}\right]
$$

is minimized at each time point over all possible values of the unknown noise covariance $W_{k}$ subject to the probability constraint (5), and a suboptimal filter is obtained eventually.

In short, it is our aim in this paper to seek a suboptimal filtering algorithm via solving the following

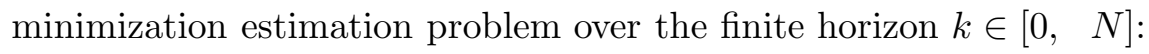

$$
\begin{gathered}
J_{k}^{\mathrm{opt}}=\inf _{\mathscr{F}} \sup _{W_{k}} J\left(\mathscr{F} ; W_{k}\right) \\
\text { subject to: } \quad W_{k} \in \mathcal{W}, \quad \operatorname{Prob}\left\{z_{k} \leq \varphi_{k}\right\} \geq \gamma_{k} .
\end{gathered}
$$

Now, we shall recall a lemma which is useful for the subsequent derivation.

Lemma 1: [29] Consider system (1) and the filter $\mathscr{F}$ of the form (6). If the noise covariance $W_{k}$ belongs to a compact convex set $\mathcal{W}$, then there exists a $J_{k}^{\text {opt }}$ such that

$$
\inf _{\mathscr{F}} \sup _{W_{k}} J_{k}\left(\mathscr{F} ; W_{k}\right)=J_{k}^{\mathrm{opt}}=\sup _{W_{k}} \inf _{\mathscr{F}} J_{k}\left(\mathscr{F} ; W_{k}\right) .
$$

With the benefit of Lemma 1, the required suboptimal filter can be determined via the procedure stated as follows:

Step 1. At each time point, find the upper-bound $Q_{k}$ on the state estimation error covariance in the presence of parameter uncertainty, measurements degradation and nonlinear stochastic disturbance occurring during the process of measurement collecting; 
Step 2. Solve the following optimization problem:

$$
\tilde{J}_{k}=\inf _{\mathscr{F}} J_{k}\left(\mathscr{F} ; W_{k}\right),
$$

to determine the parameters $A_{k f}$ and $K_{k f}$ capable of minimizing the performance index defined by cost function (8) at each time point. In this step, we could obtain the parametric expressions of filter parameters $A_{k f}$ and $K_{k f}$ in terms of $W_{k}$;

Step 3. Obtain the ultimate suboptimal filter via solving the optimization problem:

$$
J_{k}^{\mathrm{opt}}=\sup _{W_{k}} \tilde{J}_{k}=\sup _{W_{k}} \inf _{\mathscr{F}} J_{k}\left(\mathscr{F} ; W_{k}\right),
$$

by incorporating the additional knowledge on $W_{k}$ subject to probability constraint (5), and then obtain the desired filter parameters.

Generally speaking, in this paper, we shall try to design a filter $\mathscr{F}$ using the output measurement $y_{k}$ while taking advantage of the additional knowledge of the bounded measurement output $z_{k}$ subject to the probability constraint (5) with the hope of reducing the conservatism of the proposed minimization approach, thereby improving the estimation accuracy. This problem will be referred to as a probability constrained finite-horizon robust filtering problem.

\section{Finite-Horizon Filter Design}

In this section, a robust filter design problem for the discrete nonlinear stochastic system (1) will be discussed over the finite horizon. Specifically, we shall proceed to deal with Step 1 and Step 2 of the design procedures for the required suboptimal filter mentioned in the previous section.

First of all, by defining a new vector as $\xi_{k} \triangleq\left[\begin{array}{ll}x_{k}^{\mathrm{T}} & \hat{x}_{k}^{\mathrm{T}}\end{array}\right]^{\mathrm{T}}$, we can obtain the following augmented system from system (1) and filter (6):

$$
\xi_{k+1}=\left(\bar{A}_{k}+\tilde{A}_{k}\right) \xi_{k}+\bar{B}_{k} \omega_{k}+\bar{G}_{k} g\left(x_{k}, k\right)
$$

where

$$
\begin{aligned}
& \bar{A}_{k}=\left[\begin{array}{cc}
A_{k} & 0 \\
K_{f k} \bar{\Theta}_{k}\left(C_{k}+\Delta C_{k}\right) & A_{f k}-K_{f k} \bar{\Theta}_{k} C_{k}
\end{array}\right], \quad \tilde{A}_{k}=\left[\begin{array}{cc}
0 & 0 \\
K_{f k} \tilde{\Theta}_{k}\left(C_{k}+\Delta C_{k}\right) & 0
\end{array}\right], \\
& \bar{B}_{k}=\left[\begin{array}{c}
B_{k} \\
K_{f k} D_{k}
\end{array}\right], \quad \bar{G}_{k}=\left[\begin{array}{c}
0 \\
K_{f k} G_{k}
\end{array}\right], \quad \tilde{\Theta}_{k}=\Theta_{k}-\bar{\Theta}_{k} .
\end{aligned}
$$

Define the following second moment for the augmented system (13):

$$
\tilde{\Xi}_{k} \triangleq \mathbb{E}\left\{\xi_{k} \xi_{k}^{\mathrm{T}}\right\}=\mathbb{E}\left\{\left[\begin{array}{c}
x_{k} \\
\hat{x}_{k}
\end{array}\right]\left[\begin{array}{c}
x_{k} \\
\hat{x}_{k}
\end{array}\right]^{\mathrm{T}}\right\} .
$$

According to (13) and (14), we could obtain a Lyapunov equation that governs the evolution of $\tilde{\Xi}_{k+1}$ as follows:

$$
\tilde{\Xi}_{k+1}=\mathbb{E}\left\{\left(\left(\bar{A}_{k}+\tilde{A}_{k}\right) \xi_{k}+\bar{B}_{k} \omega_{k}+\bar{G}_{k} g\left(x_{k}, k\right)\right)\left(\left(\bar{A}_{k}+\tilde{A}_{k}\right) \xi_{k}+\bar{B}_{k} \omega_{k}+\bar{G}_{k} g\left(x_{k}, k\right)\right)^{\mathrm{T}}\right\} .
$$

Taking the statistical properties of stochastic nonlinear function $g\left(x_{k}, k\right)$ and white noise $\omega_{k}$ into consideration, we shall have

$$
\begin{aligned}
\tilde{\Xi}_{k+1} & =\mathbb{E}\left\{\left(\bar{A}_{k}+\tilde{A}_{k}\right) \xi_{k} \xi_{k}^{\mathrm{T}}\left(\bar{A}_{k}+\tilde{A}_{k}\right)^{\mathrm{T}}\right\}+\mathbb{E}\left\{\bar{B}_{k} \omega_{k} \omega_{k}^{\mathrm{T}} \bar{B}_{k}^{\mathrm{T}}\right\}+\mathbb{E}\left\{\bar{G}_{k} g\left(x_{k}, k\right) g^{\mathrm{T}}\left(x_{k}, k\right) \bar{G}_{k}^{\mathrm{T}}\right\} \\
& =\bar{A}_{k} \tilde{\Xi}_{k} \bar{A}_{k}^{\mathrm{T}}+\sum_{j=1}^{m} \sigma_{j k}^{2} \bar{C}_{j k} \tilde{\Xi}_{k} \bar{C}_{j k}^{\mathrm{T}}+\bar{B}_{k} W_{k} \bar{B}_{k}^{\mathrm{T}}+\bar{G}_{k} \sum_{i=1}^{q} \Pi_{i k} \operatorname{tr}\left[\Gamma_{i k} P_{k}\right] \bar{G}_{k}^{\mathrm{T}},
\end{aligned}
$$


where

$$
P_{k}=\mathbb{E}\left\{x_{k} x_{k}^{\mathrm{T}}\right\}, \quad \bar{C}_{j k}=\left[\begin{array}{cc}
0 & 0 \\
K_{f k} e_{j}\left(C_{k}+\Delta C_{k}\right) & 0
\end{array}\right], \quad e_{j}=\operatorname{diag}\{\underbrace{0, \ldots, 0}_{j-1}, 1, \underbrace{0, \ldots, 0}_{m-j}\} .
$$

It is worth noting that the parameter uncertainty $\Delta C_{k}$ is involved in equation (16) which hinders us from obtaining the exact value of the matrix $\tilde{\Xi}_{k}$. Consequently, in the following stage, we shall proceed to propose an algorithm to eliminate the parameter uncertainty $\Delta C_{k}$, and then give an alternative way by which a set of upper-bounds for $\tilde{\Xi}_{k}$ can be guaranteed. To this end, a useful lemma that is capable of dealing with the parameter uncertainty is firstly introduced.

Lemma 2: [13] Given matrices $A, H, E$, and $F$ with compatible dimensions such that $F F^{\mathrm{T}} \leq I$. Let $X$ be a symmetric positive definite matrix and $\alpha>0$ be an arbitrary positive constant such that $\alpha^{-1} I-E X E^{\mathrm{T}}>0$, then the following inequality holds

$$
(A+H F E) X(A+H F E)^{\mathrm{T}} \leq A\left(X^{-1}-\alpha E^{\mathrm{T}} E\right)^{-1} A^{\mathrm{T}}+\alpha^{-1} H H^{\mathrm{T}} .
$$

Next, in order to eliminate the parameter uncertainty, we rewrite the uncertain terms in (16) as follows:

$$
\begin{gathered}
\bar{A}_{k} \tilde{\Xi}_{k} \bar{A}_{k}^{\mathrm{T}}=\left(\hat{A}_{k}+\tilde{H}_{1 k} F_{k} \tilde{E}_{k}\right) \tilde{\Xi}_{k}\left(\hat{A}_{k}+\tilde{H}_{1 k} F_{k} \tilde{E}_{k}\right)^{\mathrm{T}} \\
\sum_{j=1}^{m} \sigma_{j k}^{2} \bar{C}_{j k} \tilde{\Xi}_{k} \bar{C}_{j k}^{\mathrm{T}}=\sum_{j=1}^{m} \sigma_{j k}^{2}\left(\hat{C}_{j k}+\tilde{H}_{2 k} F_{k} \tilde{E}_{k}\right) \tilde{\Xi}_{k}\left(\hat{C}_{j k}+\tilde{H}_{2 k} F_{k} \tilde{E}_{k}\right)^{\mathrm{T}}
\end{gathered}
$$

where

$$
\begin{aligned}
& \hat{A}_{k}=\left[\begin{array}{cc}
A_{k} & 0 \\
K_{f k} \bar{\Theta}_{k} C_{k} & A_{f k}-K_{f k} \bar{\Theta}_{k} C_{k}
\end{array}\right], \quad \hat{C}_{j k}=\left[\begin{array}{cc}
0 & 0 \\
K_{f k} e_{j} C_{k} & 0
\end{array}\right] \text {, } \\
& \tilde{H}_{1 k}=\left[\begin{array}{c}
0 \\
K_{f k} \bar{\Theta}_{k} H_{k}
\end{array}\right], \quad \tilde{H}_{2 k}=\left[\begin{array}{c}
0 \\
K_{f k} e_{j} H_{k}
\end{array}\right], \quad \tilde{E}_{k}=\left[\begin{array}{ll}
E_{k} & 0
\end{array}\right] \text {. }
\end{aligned}
$$

It follows from Lemma 2 that, if there exists $\alpha_{k}>0$ such that $\alpha_{k}^{-1} I-\tilde{E}_{k} \tilde{\Xi}_{k} \tilde{E}^{\mathrm{T}}>0$, then the following matrix inequalities hold:

$$
\begin{gathered}
\bar{A}_{k} \tilde{\Xi}_{k} \bar{A}_{k}^{\mathrm{T}} \leq \hat{A}_{k}\left(\tilde{\Xi}_{k}^{-1}-\alpha_{k} \tilde{E}_{k}^{\mathrm{T}} \tilde{E}_{k}\right)^{-1} \hat{A}_{k}^{\mathrm{T}}+\alpha_{k}^{-1} \tilde{H}_{1 k} \tilde{H}_{1 k}^{\mathrm{T}} \\
\sum_{j=1}^{m} \sigma_{j k}^{2} \bar{C}_{j k} \tilde{\Xi}_{k} \bar{C}_{j k}^{\mathrm{T}} \leq \sum_{j=1}^{m} \sigma_{j k}^{2}\left(\hat{C}_{j k}\left(\tilde{\Xi}_{k}^{-1}-\alpha_{k} \tilde{E}_{k}^{\mathrm{T}} \tilde{E}_{k}\right)^{-1} \hat{C}_{j k}^{\mathrm{T}}+\alpha_{k}^{-1} \tilde{H}_{2 k} \tilde{H}_{2 k}^{\mathrm{T}}\right) .
\end{gathered}
$$

Therefore, we can conclude that the following matrix inequality of $\tilde{\Xi}_{k}$ is satisfied:

$$
\begin{aligned}
\tilde{\Xi}_{k+1} \leq & \hat{A}_{k}\left(\tilde{\Xi}_{k}^{-1}-\alpha_{k} \tilde{E}_{k}^{\mathrm{T}} \tilde{E}_{k}\right)^{-1} \hat{A}_{k}^{\mathrm{T}}+\alpha_{k}^{-1} \tilde{H}_{1 k} \tilde{H}_{1 k}^{\mathrm{T}} \\
& +\sum_{j=1}^{m} \sigma_{j k}^{2}\left(\hat{C}_{j k}\left(\tilde{\Xi}_{k}^{-1}-\alpha_{k} \tilde{E}_{k}^{\mathrm{T}} \tilde{E}_{k}\right)^{-1} \hat{C}_{j k}^{\mathrm{T}}+\alpha_{k}^{-1} \tilde{H}_{2 k} \tilde{H}_{2 k}^{\mathrm{T}}\right) \\
& +\bar{B}_{k} W_{k} \bar{B}_{k}^{\mathrm{T}}+\bar{G}_{k} \sum_{i=1}^{q} \Pi_{i k} \operatorname{tr}\left[\Gamma_{i k} P_{k}\right] \bar{G}_{k}^{\mathrm{T}} .
\end{aligned}
$$

Up to now, we have eliminated the parameter uncertainty $\Delta C_{k}$ by means of the technique introduced by Lemma 2. We now turn to seek the upper-bound of $\tilde{\Xi}_{k}$ defined in (14). We will show that it can be found via solving a set of Riccati-like difference equations at corresponding time point. Before giving the detailed design technique, a useful lemma is introduced. 
Lemma 3: [13] For $0 \leq k \leq N$, suppose that $X=X^{\mathrm{T}}>0$, and $f_{k}(X)=f_{k}^{\mathrm{T}}(X), h_{k}(X)=h_{k}^{\mathrm{T}}(X)$, where $f_{k}(\cdot)$ and $h_{k}(\cdot)$ are matrix-valued functionals. If there exists $Y=Y^{\mathrm{T}}>X$ such that

$$
f_{k}(Y) \geq f_{k}(X)
$$

and

$$
h_{k}(Y) \geq f_{k}(Y),
$$

then the solutions $X_{k}$ and $Y_{k}$ to the following difference equations

$$
X_{k+1}=f_{k}\left(X_{k}\right), \quad Y_{k+1}=h_{k}\left(Y_{k}\right), \quad X_{0}=Y_{0}
$$

satisfy $X_{k} \leq Y_{k}$.

Construct a matrix-valued difference equation as:

$$
\begin{aligned}
\Xi_{k+1} \triangleq & \hat{A}_{k}\left(\Xi_{k}^{-1}-\alpha_{k} \tilde{E}_{k}^{\mathrm{T}} \tilde{E}_{k}\right)^{-1} \hat{A}_{k}^{\mathrm{T}}+\alpha_{k}^{-1} \tilde{H}_{1 k} \tilde{H}_{1 k}^{\mathrm{T}} \\
& +\sum_{j=1}^{m} \sigma_{j k}^{2}\left(\hat{C}_{j k}\left(\Xi_{k}^{-1}-\alpha_{k} \tilde{E}_{k}^{\mathrm{T}} \tilde{E}_{k}\right)^{-1} \hat{C}_{j k}^{\mathrm{T}}+\alpha_{k}^{-1} \tilde{H}_{2 k} \tilde{H}_{2 k}^{\mathrm{T}}\right) \\
& +\bar{B}_{k} W_{k} \bar{B}_{k}^{\mathrm{T}}+\bar{G}_{k} \sum_{i=1}^{q} \Pi_{i k} \operatorname{tr}\left[\Gamma_{i k} P_{k}\right] \bar{G}_{k}^{\mathrm{T}}
\end{aligned}
$$

and a matrix inequality as:

$$
\alpha_{k}^{-1} I-\tilde{E}_{k} \Xi_{k} \tilde{E}^{\mathrm{T}}>0
$$

with some positive scalars $\alpha_{k}>0$ and the initial condition $\Xi_{0}=\tilde{\Xi}_{0}$. According to (22), (26), (27), and based on Lemma 3, the following theorem gives a conclusion that the solution $\Xi_{k}$ to the difference equation (26) and matrix inequality (27) provide an upper-bound on $\tilde{\Xi}_{k}$ defined in (15).

Theorem 1: Given $\tilde{\Xi}_{k}$ satisfying equation (15), $\Xi_{k}$ satisfying equation (26) and inequality (27). If the initial condition satisfies $\tilde{\Xi}_{0}=\Xi_{0}$, then $\tilde{\Xi}_{k} \leq \Xi_{k}$ holds. In other words, $\Xi_{k}$ is the upper-bound on $\tilde{\Xi}_{k}$.

Proof: We define two matrix equations from (15) and (26) as follows:

$$
\begin{aligned}
f_{k}\left(\tilde{\Xi}_{k}\right) \triangleq & \bar{A}_{k} \tilde{\Xi}_{k} \bar{A}_{k}^{\mathrm{T}}+\sum_{j=1}^{m} \sigma_{j k}^{2} \bar{C}_{j k} \tilde{\Xi}_{k} \bar{C}_{j k}^{\mathrm{T}}+\bar{B}_{k} W_{k} \bar{B}_{k}^{\mathrm{T}}+\bar{G}_{k} \sum_{i=1}^{q} \Pi_{i k} \operatorname{tr}\left[\Gamma_{i k} P_{k}\right] \bar{G}_{k}^{\mathrm{T}}, \\
h_{k}\left(\Xi_{k}\right) \triangleq & \hat{A}_{k}\left(\Xi_{k}^{-1}-\alpha_{k} \tilde{E}_{k}^{\mathrm{T}} \tilde{E}_{k}\right)^{-1} \hat{A}_{k}^{\mathrm{T}}+\alpha_{k}^{-1} \tilde{H}_{1 k} \tilde{H}_{1 k}^{\mathrm{T}} \\
& +\sum_{j=1}^{m} \sigma_{j k}^{2}\left(\hat{C}_{j k}\left(\Xi_{k}^{-1}-\alpha_{k} \tilde{E}_{k}^{\mathrm{T}} \tilde{E}_{k}\right)^{-1} \hat{C}_{j k}^{\mathrm{T}}+\alpha_{k}^{-1} \tilde{H}_{2 k} \tilde{H}_{2 k}^{\mathrm{T}}\right) \\
& +\bar{B}_{k} W_{k} \bar{B}_{k}^{\mathrm{T}}+\bar{G}_{k} \sum_{i=1}^{q} \Pi_{i k} \operatorname{tr}\left[\Gamma_{i k} P_{k}\right] \bar{G}_{k}^{\mathrm{T}} .
\end{aligned}
$$

Then it can be easily checked from Lemma 3 that $\tilde{\Xi}_{k} \leq \Xi_{k}$ holds provided initial condition $\tilde{\Xi}_{0}=\Xi_{0}$.

Remark 4: Theorem 1 shows that the upper-bound on $\tilde{\Xi}_{k}$ defined in (14) for the augmented system (13) can be guaranteed in terms of the solution $\Xi_{k}$ to the equation (26) with inequality (27). It is worth mentioning that such solutions are not unique generally. In the following, an attempt will be made to solve (26) and (27) to select the filter parameters $A_{f k}$ and $K_{f k}$ so that the obtained upper-bound is minimized. 
Theorem 2: Consider system (1). Let $\alpha_{k}>0$ be a sequence of positive scalars satisfying $\alpha_{k}^{-1} I-E_{k} P_{k} E_{k}^{\mathrm{T}}>0$. If the following set of matrix-valued difference equations:

$$
\begin{gathered}
P_{k+1}=A_{k} P_{k} A_{k}^{\mathrm{T}}+B_{k} W_{k} B_{k}^{\mathrm{T}} \\
Q_{k+1}=-\left(A_{k}\left(Q_{k}^{-1}-\alpha_{k} E_{k}^{\mathrm{T}} E_{k}\right)^{-1} C_{k}^{\mathrm{T}} \bar{\Theta}_{k}+B_{k} W_{k} D_{k}^{\mathrm{T}}\right) \Omega_{k}^{-1} \\
\times\left(A_{k}\left(Q_{k}^{-1}-\alpha_{k} E_{k}^{\mathrm{T}} E_{k}\right)^{-1} C_{k}^{\mathrm{T}} \bar{\Theta}_{k}+B_{k} W_{k} D_{k}^{\mathrm{T}}\right)^{\mathrm{T}} \\
+A_{k}\left(Q_{k}^{-1}-\alpha_{k} E_{k}^{\mathrm{T}} E_{k}\right)^{-1} A_{k}^{\mathrm{T}}+B_{k} W_{k} B_{k}^{\mathrm{T}},
\end{gathered}
$$

where

$$
\begin{aligned}
\Omega_{k}= & \bar{\Theta}_{k} C_{k}\left(Q_{k}^{-1}-\alpha_{k} E_{k}^{\mathrm{T}} E_{k}\right)^{-1} C_{k}^{\mathrm{T}} \bar{\Theta}_{k}+\alpha_{k}^{-1} \bar{\Theta}_{k} H_{k} H_{k}^{\mathrm{T}} \bar{\Theta}_{k} \\
& +\sum_{j=1}^{m} \sigma_{j k}^{2}\left(e_{j} C_{k}\left(Q_{k}^{-1}-\alpha_{k} E_{k}^{\mathrm{T}} E_{k}\right)^{-1} C_{k}^{\mathrm{T}} e_{j}+\alpha_{k}^{-1} e_{j} H_{k} H_{k}^{\mathrm{T}} e_{j}\right) \\
& +\sum_{i=1}^{q} G_{k} \Pi_{i k} \operatorname{tr}\left[\Gamma_{i k} P_{k}\right] G_{k}^{\mathrm{T}}+D_{k} W_{k} D_{k}^{\mathrm{T}},
\end{aligned}
$$

have positive definite solutions $P_{k}$ and $Q_{k}$ such that $P_{k}-Q_{k}>0$, then there exists a required filter with the parameters

$$
A_{f k}=A_{k}+\left(A_{k}-K_{f k} \bar{\Theta}_{k} C_{k}\right) Q_{k} E_{k}^{\mathrm{T}}\left(\alpha_{k}^{-1} I-E_{k} Q_{k} E_{k}^{\mathrm{T}}\right)^{-1} E_{k}
$$

and

$$
K_{f k}=\left(B_{k} W_{k} D_{k}^{\mathrm{T}}+A_{k}\left(Q_{k}^{-1}-\alpha_{k} E_{k}^{\mathrm{T}} E_{k}\right)^{-1} C_{k}^{\mathrm{T}} \bar{\Theta}_{k}\right) \Omega_{k}^{-1},
$$

such that the state estimation error covariance satisfies

$$
\mathbb{E}\left\{\left(x_{k}-\hat{x}_{k}\right)\left(x_{k}-\hat{x}_{k}\right)^{\mathrm{T}}\right\} \leq Q_{k}
$$

In other words, $Q_{k}$ is the upper-bound of the estimation error covariance. Moreover, with the obtained filter parameters $A_{f k}$ and $K_{f k}$, such an upper-bound $Q_{k}$ is minimized in the sense of matrix norm, which indicates that the minimum of $\tilde{J}_{k}$ defined in (11) is guaranteed.

Proof: First, assume that $\Xi_{k}$ can be decomposed as follows:

$$
\Xi_{k}=\left[\begin{array}{cc}
P_{k} & P_{k}-Q_{k} \\
P_{k}-Q_{k} & P_{k}-Q_{k}
\end{array}\right]
$$

where $P_{k}$ and $Q_{k}$ are defined in (29) and (30) respectively.

Next, we shall show that $\Xi_{k}$ defined in (35) is a solution to (26). To this end, substituting the filter parameters $A_{f k}$ and $K_{f k}$ in (32) and (33) into $P_{k+1}$ of (29) and $Q_{k+1}$ of (30) respectively, after some tedious but straightforward manipulations, we can find out that $\Xi_{k}$ is a solution to the matrix-valued function (26). Therefore, according to Theorem $1, \Xi_{k}$ is the upper-bound of the covariance of system (13). The rest of the proof is to show the obtained $A_{f k}$ and $K_{f k}$ can minimize the estimation error covariance upper-bound $Q_{k}$. 
To this end, it is obvious to see that

$$
\begin{aligned}
Q_{k+1}= & {\left[\begin{array}{ll}
I & -I
\end{array}\right] \Xi_{k+1}\left[\begin{array}{ll}
I & -I
\end{array}\right]^{\mathrm{T}} } \\
= & {\left[\begin{array}{ll}
A_{k}-K_{f k} \bar{\Theta}_{k} C_{k} & -A_{f k}-K_{f k} \bar{\Theta}_{k} C_{k}
\end{array}\right]\left(\Xi_{k}^{-1}-\alpha_{k} \tilde{E}_{k}^{\mathrm{T}} \tilde{E}_{k}\right)^{-1} } \\
& \times\left[\begin{array}{ll}
A_{k}-K_{f k} \bar{\Theta}_{k} C_{k} & -A_{f k}-K_{f k} \bar{\Theta}_{k} C_{k}
\end{array}\right]^{\mathrm{T}} \\
& +\sum_{j=1}^{m} \sigma_{j k}^{2}\left[\begin{array}{ll}
-K_{f k} e_{j} C_{k} & 0
\end{array}\right]\left(\Xi_{k}^{-1}-\alpha_{k} \tilde{E}_{k}^{\mathrm{T}} \tilde{E}_{k}\right)^{-1}\left[\begin{array}{ll}
-K_{f k} e_{j} C_{k} & 0
\end{array}\right]^{\mathrm{T}} \\
& +\sum_{j=1}^{m} \sigma_{j k}^{2} \alpha_{k}^{-1} K_{f k} e_{j} H_{k} H_{k}^{\mathrm{T}} e_{j} K_{f k}^{\mathrm{T}}+\alpha_{k}^{-1} K_{f k} \bar{\Theta}_{k} H_{k} H_{k}^{\mathrm{T}} \bar{\Theta}_{k} K_{f k}^{\mathrm{T}} \\
& +\left(B_{k}-K_{f k} D_{k}\right) W_{k}\left(B_{k}-K_{f k} D_{k}\right)^{\mathrm{T}}+\sum_{i=1}^{q} K_{f k} G_{k} \Pi_{i k} \operatorname{tr}\left[\Gamma_{i k} P_{k}\right] G_{k}^{\mathrm{T}} K_{f k}^{\mathrm{T}} .
\end{aligned}
$$

In order to find out the filter parameters minimizing the upper-bound of estimation error variance $Q_{k}$ at each time point $k$, we take the first variation of (36) with respective to $A_{f k}$ and $K_{f k}$ as follows:

$$
\begin{aligned}
& \frac{\partial Q_{k+1}}{\partial A_{f k}}=2\left[\begin{array}{ll}
A_{k}-K_{f k} \bar{\Theta}_{k} C_{k} & -A_{f k}-K_{f k} \bar{\Theta}_{k} C_{k}
\end{array}\right] \\
& \times\left(\Xi_{k}^{-1}-\alpha_{k} \tilde{E}_{k}^{\mathrm{T}} \tilde{E}_{k}\right)^{-1}\left[\begin{array}{ll}
0 & -I
\end{array}\right]=0 \\
& \frac{\partial Q_{k+1}}{\partial K_{f k}}=2\left[\begin{array}{ll}
A_{k}-K_{f k} \bar{\Theta}_{k} C_{k} & -A_{f k}-K_{f k} \bar{\Theta}_{k} C_{k}
\end{array}\right] \\
& \times\left(\Xi_{k}^{-1}-\alpha_{k} \tilde{E}_{k}^{\mathrm{T}} \tilde{E}_{k}\right)^{-1}\left[\begin{array}{ll}
-\bar{\Theta}_{k} C_{k} & \bar{\Theta}_{k} C_{k}
\end{array}\right]^{\mathrm{T}} \\
&+2 \sum_{j=1}^{m} \sigma_{j k}^{2}\left[-K_{f k} e_{j} C_{k} \quad 0\right]\left(\Xi_{k}^{-1}-\alpha_{k} \tilde{E}_{k}^{\mathrm{T}} \tilde{E}_{k}\right)^{-1}\left[\begin{array}{ll}
-e_{j} C_{k} & 0
\end{array}\right]^{\mathrm{T}} \\
&+2 \sum_{j=1}^{m} \sigma_{j k}^{2} \alpha_{k}^{-1} K_{f k} e_{j} H_{k} H_{k}^{\mathrm{T}} e_{j}+2 \alpha_{k}^{-1} K_{f k} \bar{\Theta}_{k} H_{k} H_{k}^{\mathrm{T}} \bar{\Theta}_{k} \\
&+2\left(B_{k}-K_{f k} D_{k}\right) W_{k} D_{k}^{\mathrm{T}}+2 \sum_{i=1}^{q} K_{f k} G_{k} \Pi_{i k} \operatorname{tr}\left[\Gamma_{i k} P_{k}\right] G_{k}^{\mathrm{T}}=0 .
\end{aligned}
$$

Then, $A_{f k}$ can be obtained by

$$
\begin{aligned}
A_{f k}= & A_{k}+\left(A_{k}-K_{f k} \bar{\Theta}_{k} C_{k}\right) Q_{k} E_{k}^{\mathrm{T}}\left(\alpha_{k}^{-1} I-E_{k} P_{k} E_{k}^{\mathrm{T}}\right)^{-1} E_{k} \\
& \times\left(I-\left(Q_{k}-P_{k}\right) E_{k}^{\mathrm{T}}\left(\alpha_{k}^{-1} I-E_{k} P_{k} E_{k}^{\mathrm{T}}\right)^{-1} E_{k}\right)^{-1} \\
= & A_{k}+\left(A_{k}-K_{f k} \bar{\Theta}_{k} C_{k}\right) Q_{k} E_{k}^{\mathrm{T}} E_{k}\left(\alpha_{k}^{-1} I-P_{k} E_{k}^{\mathrm{T}} E_{k}\right)^{-1} \\
& \times\left(I-\left(Q_{k}-P_{k}\right) E_{k}^{\mathrm{T}} E_{k}\left(\alpha_{k}^{-1} I-E_{k} P_{k} E_{k}^{\mathrm{T}}\right)^{-1}\right)^{-1} \\
= & A_{k}+\left(A_{k}-K_{f k} \bar{\Theta}_{k} C_{k}\right) Q_{k} E_{k}^{\mathrm{T}} E_{k}\left(\alpha_{k}^{-1} I-Q_{k} E_{k}^{\mathrm{T}} E_{k}\right)^{-1} \\
= & A_{k}+\left(A_{k}-K_{f k} \bar{\Theta}_{k} C_{k}\right) Q_{k} E_{k}^{\mathrm{T}}\left(\alpha_{k}^{-1} I-E_{k} Q_{k} E_{k}^{\mathrm{T}}\right)^{-1} E_{k},
\end{aligned}
$$

which is exactly the form in (32).

Likewise, by some tedious but straightforward calculations, we can find the parametric expression of parameter $K_{f k}$ as follows:

$$
K_{f k}=\left(B_{k} W_{k} D_{k}^{\mathrm{T}}+A_{k}\left(Q_{k}^{-1}-\alpha_{k} E_{k}^{\mathrm{T}} E_{k}\right)^{-1} C_{k}^{\mathrm{T}} \bar{\Theta}_{k}\right) \Omega_{k}^{-1},
$$


where

$$
\begin{aligned}
\Omega_{k}= & \bar{\Theta}_{k} C_{k}\left(Q_{k}^{-1}-\alpha_{k} E_{k}^{\mathrm{T}} E_{k}\right)^{-1} C_{k}^{\mathrm{T}} \bar{\Theta}_{k}+\alpha_{k}^{-1} \bar{\Theta}_{k} H_{k} H_{k}^{\mathrm{T}} \bar{\Theta}_{k} \\
& +\sum_{j=1}^{m} \sigma_{j k}^{2}\left(e_{j} C_{k}\left(Q_{k}^{-1}-\alpha_{k} E_{k}^{\mathrm{T}} E_{k}\right)^{-1} C_{k}^{\mathrm{T}} e_{j}+\alpha_{k}^{-1} e_{j} H_{k} H_{k}^{\mathrm{T}} e_{j}\right) \\
& +\sum_{i=1}^{q} G_{k} \Pi_{i k} \operatorname{tr}\left[\Gamma_{i k} P_{k}\right] G_{k}^{\mathrm{T}}+D_{k} W_{k} D_{k}^{\mathrm{T}} .
\end{aligned}
$$

It can be easily seen that $K_{f k}$ is the same as that in (33). The proof is complete.

Up to now, we have completed the design procedure Step 1 and Step 2 and given a sufficient condition of the existence of the required filter capable of minimizing the state estimation error covariance upper-bound in terms of the solvability of certain Riccati-like difference equations.

\section{Filter Design with Probability Constraints}

In this section, we shall proceed to reduce the conservatism of the results obtained in the previous sections by incorporating the probability constraint (5) into the optimization problem. By resorting to the technique developed in [29], the probability constraint can be converted to certain linear matrix inequalities. To this end, observe system (1) and let $P_{k}$ and $r_{k}$ denote the unique solutions to

$$
\left\{\begin{array}{l}
P_{k+1}=A_{k} P_{k} A_{k}^{\mathrm{T}}+B_{k} W_{k} B_{k}^{\mathrm{T}}, \\
r_{k+1}=A_{k} r_{k},
\end{array}\right.
$$

with initial conditions $P_{0}=X_{0}$ and $r_{0}=\bar{x}_{0}$, respectively.

Denote the $i$ th entry of the probability bound vector $\gamma_{k}$ as $\gamma_{i, k}$ and the $i$ th entry of $\varphi_{k}$ as $\varphi_{i, k}$. Denote the $i$ th line of matrices $L_{k}$ and $M_{k}$ as $l_{i, k}$ and $m_{i, k}$, respectively. Then the $i$ th entry of measurement $z_{k}$ can be represented as follows:

$$
z_{i, k}=l_{i, k} x_{k}+m_{i, k} \omega_{k}
$$

Obviously, the probability constraint (5) is equivalent to

$$
\operatorname{Prob}\left\{l_{i, k} x_{k}+m_{i, k} \omega_{k} \leq \varphi_{i, k}\right\} \geq \gamma_{i, k}, \quad i=1,2, \ldots, m
$$

In the following, a lemma is given to convert the probability constraints into certain linear matrix inequalities.

Lemma 4: [29]Consider system (1). Let $\gamma_{i, k}>1 / 2$ be given and assume that $\tau_{i, k}^{*}$ is the unique solution to

Then, the probability constraint

$$
\frac{1}{\sqrt{2 \pi}} \int_{-\infty}^{\tau_{i, k}^{*}} e^{-\frac{\varsigma^{2}}{2}} d \varsigma=\gamma_{i, k} .
$$

$$
\operatorname{Prob}\left\{z_{k} \leq \varphi_{k}\right\} \geq \gamma_{k}
$$

is satisfied if and only if

$$
\left\{\begin{array}{l}
\varphi_{i, k}-l_{i, k} r_{k} \geq 0, \\
l_{i, k} P_{k} l_{i, k}^{\mathrm{T}}+m_{i, k} W_{k} m_{i, k}^{\mathrm{T}} \leq\left(\frac{\varphi_{i, k}-l_{i, k} r_{k}}{\tau_{i, k}^{*}}\right)^{2}, \quad i=1,2, \ldots, m
\end{array}\right.
$$

where $P_{k}$ and $r_{k}$ are the solutions of difference matrix equations (42). 
Proof: It is easy to know that $z_{i, k}$ is a random variable with mean and covariance being governed by

$$
\begin{aligned}
\mathbb{E}\left\{z_{i, k}\right\} & =l_{i, k} r_{k}, \\
\mathbb{E}\left\{\left(z_{i, k}-l_{i, k} r_{k}\right)\left(z_{i, k}-l_{i, k} r_{k}\right)^{\mathrm{T}}\right\} & =l_{i, k} P_{k} l_{i, k}^{\mathrm{T}}+m_{i, k} W_{k} m_{i, k}^{\mathrm{T}},
\end{aligned}
$$

where $P_{k}$ and $r_{k}$ are the solutions of difference matrix equations (42).

Define now

$$
\tau_{i, k}=\frac{\varphi_{i, k}-l_{i, k} r_{k}}{\sqrt{l_{i, k} P_{k} l_{i, k}^{\mathrm{T}}+m_{i, k} W_{k} m_{i, k}^{\mathrm{T}}}} .
$$

Then it is not difficult to obtain that

$$
\operatorname{Prob}\left\{l_{i, k} x_{k}+m_{i, k} \omega_{k} \leq \varphi_{i, k}\right\}=\frac{1}{\sqrt{2 \pi}} \int_{-\infty}^{\tau_{i, k}} e^{-\frac{\varsigma^{2}}{2}} d \varsigma .
$$

Notice that the left hand side of (50) is a monotone increasing function of $\tau_{i, k}$. Consequently, it is easy to know that

$$
\begin{aligned}
& \operatorname{Prob}\left\{z_{i, k} \leq \varphi_{i, k}\right\} \geq \gamma_{i, k} \\
\Longleftrightarrow & \tau_{i, k} \geq \tau_{i, k}^{*} \\
\Longleftrightarrow & l_{i, k} X_{k} l_{i, k}^{\mathrm{T}}+m_{i, k} W_{k} m_{i, k}^{\mathrm{T}} \leq\left(\frac{\varphi_{i, k}-l_{i, k} r_{k}}{\tau_{i, k}^{*}}\right)^{2} .
\end{aligned}
$$

The proof is now complete.

Based on Theorem 2 and Lemma 4, we can immediately obtain the following theorem giving a sufficient condition to solve the addressed suboptimal filtering problem for nonlinear time-varying systems with parameter uncertainty, measurements degradation and stochastic nonlinearity.

Theorem 3: Consider system (1) and the probability constraint (5) with $\varphi_{i, k}$ and $\gamma_{i, k}>1 / 2$ being given. The worst-case performance defined in (9) can be obtained by solving the following minimization problem

$$
J_{\mathrm{opt}}=\sup _{W_{k}} \inf _{\mathscr{F}} J\left(\mathscr{F} ; W_{k}\right)
$$

subject to the following constraints:

$$
\begin{aligned}
P_{k+1}= & A_{k} P_{k} A_{k}^{\mathrm{T}}+B_{k} W_{k} B_{k}^{\mathrm{T}} \\
Q_{k+1}= & -\left(A_{k}\left(Q_{k}^{-1}-\alpha_{k} E_{k}^{\mathrm{T}} E_{k}\right)^{-1} C_{k}^{\mathrm{T}} \bar{\Theta}_{k}+B_{k} W_{k} D_{k}^{\mathrm{T}}\right) \Omega_{k}^{-1} \\
& \times\left(A_{k}\left(Q_{k}^{-1}-\alpha_{k} E_{k}^{\mathrm{T}} E_{k}\right)^{-1} C_{k}^{\mathrm{T}} \bar{\Theta}_{k}+B_{k} W_{k} D_{k}^{\mathrm{T}}\right)^{\mathrm{T}} \\
& +A_{k}\left(Q_{k}^{-1}-\alpha_{k} E_{k}^{\mathrm{T}} E_{k}\right)^{-1} A_{k}^{\mathrm{T}}+B_{k} W_{k} B_{k}^{\mathrm{T}} \\
0< & P_{k}-Q_{k} \\
0< & \alpha_{k}^{-1} I-E_{k} P_{k} E_{k}^{\mathrm{T}} \\
l_{i, k} P_{k} l_{i, k}^{\mathrm{T}}+m_{i, k} W_{k} m_{i, k}^{\mathrm{T}} \leq & \left(\frac{\varphi_{i, k}-l_{i, k} r_{k}}{\tau_{i, k}^{*}}\right)^{2}, \quad i=1,2, \ldots, m
\end{aligned}
$$

Moreover, the suboptimal filtering parameters at each time point can be obtained by (32) and (33).

Proof: Based on Theorem 2 and Lemma 4, the proof of Theorem 3 is quite straightforward, and therefore is omitted here.

So far, the whole procedures of the addressed suboptimal filter design problem has been finished. The existence of the desired filter can be checked by solving the set of Riccati-like difference equations and linear 
matrix inequalities. The filter gains at each time point can be determined by solving the corresponding set of equations and inequalities provided the minimization problem is solvable. The following algorithm presents a iterative computing method to gain the desired filter parameters by solving the corresponding minimization problem.

\section{Algorithm 1: Computational Algorithm}

Step 1 Set $k=0$. Select properly the initial values $\bar{x}_{0}, P_{0}$ and $Q_{0}$.

Step 2 Obtain the range of unknown $W_{k}$ by inequalities (57) at the time step $k$ with known parameters.

Step 3 Solve the minimization problem (52) subject to constraints (53)-(56) with the range of $W_{k}$ obtained in Step 2, and calculate $P_{k+1}$ and $Q_{k+1}$. Based on this, the filter parameters at time step $k$, that is, $A_{f k}$ and $K_{f k}$ can also be computed.

Step 4 Set $k=k+1$. If $k<N_{\max }$ ( $N_{\max }$ is the maximum iterative times), then go to Step 2. Otherwise go to Step 5.

Step 5 Stop.

\section{An Illustrative Example}

In this section, we shall present a numerical illustrative example to show the effectiveness and applicability of the proposed suboptimal filtering technique. Consider the following time-varying nonlinear stochastic system

$$
\left\{\begin{aligned}
x_{k+1} & =\left(\left[\begin{array}{cc}
0 & 0.6+0.01 \sin (0.5 k) \\
0.3+0.01 \cos (0.5 k) & 0.2
\end{array}\right]\right) x_{k}+\left[\begin{array}{c}
0.8+0.01 \sin (0.5 k) \\
0.1
\end{array}\right] \omega_{k}, \\
y_{k} & =\Theta_{k}\left(\left[\begin{array}{ll}
0.1+0.05 \cos (0.4 k) & 0.5
\end{array}\right]+0.3 F_{k}\left[\begin{array}{cc}
0.5 & 0.7
\end{array}\right]\right) x_{k}+g\left(x_{k}, k\right)+0.1 \omega_{k}, \\
z_{k} & =\left[\begin{array}{ll}
1 & 1
\end{array}\right] x_{k}+\omega_{k},
\end{aligned}\right.
$$

where $\omega_{k}$ is a zero mean Gaussian white noise process whose covariance is unknown but belongs to certain range as $W_{k} \in[a, b]$ with $a>0$ and $b>0$ being known lower- and upper-bounds. $F_{k}=0.3 \sin (3 k)$ is a deterministic perturbation matrix satisfying $F_{k} F_{k}^{\mathrm{T}} \leq I$.

In addition, we assume the stochastic matrix $\Theta_{k}$ obey uniform distribution on the interval $[0,1]$. Hence, the mathematical expectation and variance can be easily calculated as $\bar{\Theta}_{k}=1 / 2$, and $\sigma_{j k}^{2}=1 / 12$.

The nonlinear function $g\left(x_{k}, k\right)$ is taken as follows:

$$
g\left(x_{k}, k\right)=\left[\begin{array}{ll}
0.2 & 0.3
\end{array}\right] x_{k} \xi_{k},
$$

where $\xi_{k}$ is zero mean Gaussian white noise process with unity covariance. Assume that $\xi_{k}$ is uncorrelated with $\omega_{k}$ and $\Theta_{k}$. Thus, the above stochastic nonlinearity satisfies

$$
\begin{aligned}
& \mathbb{E}\left\{\left[g\left(x_{k}, k\right)\right] \mid x_{k}\right\}=0, \\
& \mathbb{E}\left\{\left[g^{\mathrm{T}}\left(x_{k}, k\right)\right]\left[g\left(x_{k}, k\right)\right] \mid x_{k}\right\}=x_{k}^{\mathrm{T}}\left[\begin{array}{ll}
0.04 & 0.06 \\
0.06 & 0.09
\end{array}\right] x_{k} .
\end{aligned}
$$

It is assumed that the measured output $z_{k}$ satisfies the following probability constraint:

$$
\operatorname{Prob}\left\{z_{k} \leq 2.2\right\} \geq 0.8
$$


Set $N=200, k=0, a=2, b=8$ and choose the parameters' initial values as follows:

$$
r_{0}=\bar{x}_{0}=\left[\begin{array}{l}
0 \\
0
\end{array}\right], \quad \hat{x}_{0}=\left[\begin{array}{l}
1 \\
1
\end{array}\right], \quad P_{0}=\left[\begin{array}{ll}
1 & 1 \\
1 & 1
\end{array}\right], \quad Q_{0}=\left[\begin{array}{ll}
1 & 1 \\
1 & 0
\end{array}\right] .
$$

Solving the minimization problem (52), we could obtain the filtering parameters by equations (32) and (33). The detailed simulation results are shown in Table I and Figs. 1-3.

Table I shows the worst-case performance index $J_{k}^{\text {opt }}$ calculated based on Theorem 3 , where the second/third columns are the values of $J_{k}^{\text {opt }}$ obtained from the design without/with consideration of probability constraint, respectively. It should be noted that the last column shows a decrease rate of around $47 \%$ on $J_{k}^{\text {opt }}$ when the probability constraint (5) is taken into account. The plots of upper-bounds as well as the actual variances for the states $x_{k}^{1}$ and $x_{k}^{2}$ are given in Figs. 1 and 2. It can be seen, obviously, the actual variances for the states stay below their upper-bounds, which indicates that the proposed method is effective and accurate. Furthermore, the upper-bounds of each state variance in Fig. 1 are larger than those in Fig. 2, and therefore we can conclude that the obtained worst-case performance index $J_{k}^{\text {opt }}$ without probability constraint is larger than that with probability constraint, shown in Fig. 3. It proves, as we have anticipated, that the increase of system accuracy is apparent when the probability constraint is taken into consideration.

TABLE I

COMPARISON OF $J_{k}^{\text {opt }}$ IN DIFFERENT CASES

\begin{tabular}{cccc}
\hline & \multicolumn{3}{c}{$J_{k}^{\text {opt }}=\inf _{\mathscr{F}} \sup _{W_{k}} J\left(\mathcal{F} ; W_{k}\right)$} \\
\cline { 2 - 4 } time $(k)$ & Without probability constraint & With probability constraint & $J_{k}^{\text {opt }}$ \\
\hline 40 & 5.7444 & 3.0031 & $48 \%$ \\
80 & 5.6314 & 2.9942 & $48 \%$ \\
120 & 5.5113 & 2.9048 & $47 \%$ \\
160 & 5.4026 & 2.8807 & $47 \%$ \\
200 & 5.5240 & 2.9386 & $47 \%$ \\
\hline
\end{tabular}

\section{Conclusion}

This paper considers the suboptimal filtering problem for a class of time-varying systems with parameter uncertainty, measurements degradation and stochastic nonlinearity subject to probability constraint. The stochastic nonlinearity considered in this paper is quite general and could cover several classes of nonlinear stochastic systems as special cases. With the designed filtering algorithm, the upper-bound on the estimation error variance is guaranteed firstly and then is minimized in the sense of matrix norm at each time point. A minimax problem is considered to search for the worst-case criterion subject to the probability constraint on system measured output. The suboptimal filter parameters can be determined at each time point by solving the corresponding minimax problem. A numerical example is presented to show the effectiveness of the proposed filtering algorithm.

\section{REFERENCES}

[1] M. Jamshidi, H. Seraji and Y. T. Kim, Decentralized control of nonlinear robot manipulators, Robotics, Vol. 3, No. 3-4, 1987, pp. 361-370.

[2] J. Rudolph, Flatness-based control by quasi-static feedback illustrated on a cascade of two chemical reactors, International Journal of Control, Vol. 73, No. 2, 2000, pp. 115-131. 


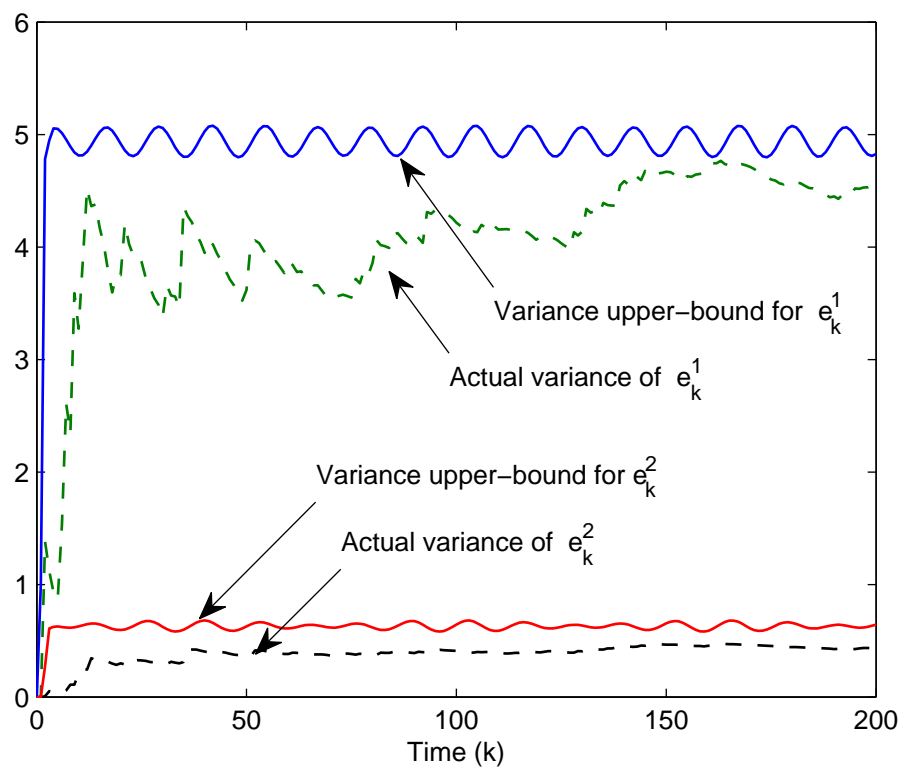

Fig. 1. The actual variances and their upper-bounds without probability constraint, where $e_{k}=x_{k}-\hat{x}_{k}$.

[3] I. R. Petersen, Robust output feedback guaranteed cost control on nonlinear stochastic systems via an IQC approach, IEEE Transactions on Automatic Control, Vol. 54, No. 6, 2009, pp. 1299-1304.

[4] H. J. Kushner, Numerical algorithms for optimal controls for nonlinear stochastic systems with delays, IEEE Transactions on Automatic Control, Vol. 55, No. 9, 2010, pp. 2170-2176.

[5] S. Khoo, J. Yin, Z. Man and X. Yu, Finite-time stabilization of stochastic nonlinear systems in strict-feedback form, Automatica, Vol. 49, No. 5, 2013, pp. 1403-1410.

[6] Y. Chen and K. A. Hoo, Stability analysis for closed-loop management of a reservoir based on identification of reduced-order nonlinear model, Systems Science and Control Engineering: An Open Access Journal, Vol. 1, No. 1, 2013, pp. 12-19.

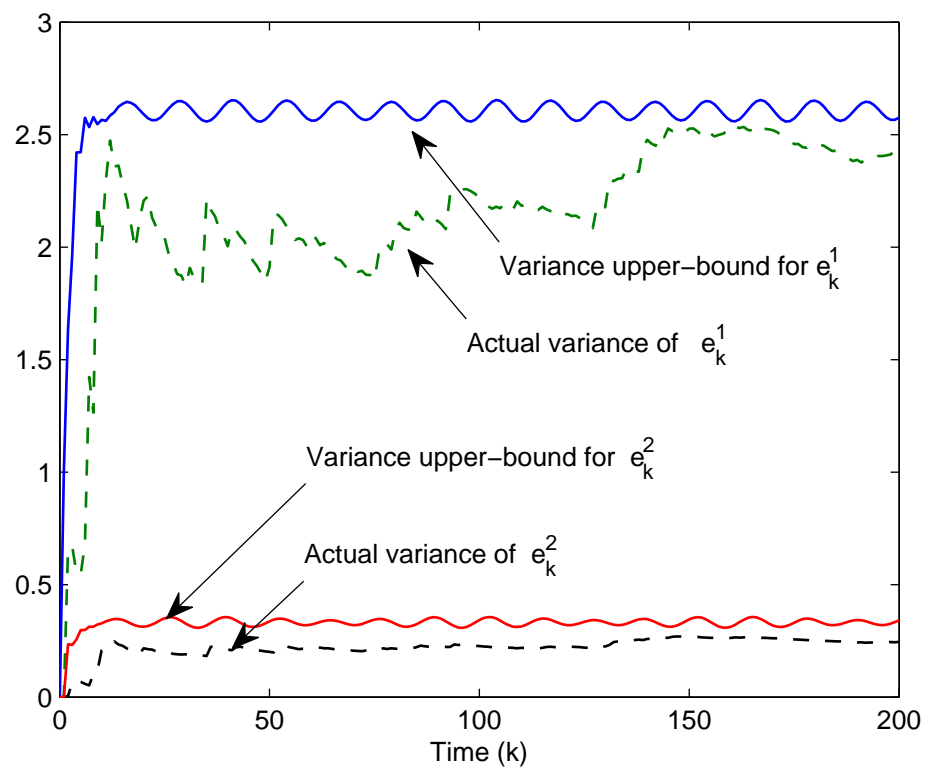

Fig. 2. The actual variances and their upper-bounds with probability constraint, where $e_{k}=x_{k}-\hat{x}_{k}$. 
[7] G. Wei, Z. Wang and B. Shen, Probability-dependent gain-scheduled control for discrete stochastic delayed systems with randomly occurring nonlinearities, International Journal of Robust and Nonlinear Control, Vol. 23, No. 7, 2013, pp. 815-826.

[8] U. Shaked and C.E. de Souza, Robust minimum variance filtering, IEEE Transactions on Signal Processing, Vol. 43, No. 11, 1995, pp. 2474-2483.

[9] B. D. Van Veen, W. van Drongelen, M. Yuchtman and A. Suzuki, Localization of brain electrical activity via linearly constrained minimum variance spatial filtering, IEEE Transactions on Biomedical Engineering, Vol. 44, No. 9, 1997, pp. 867880 .

[10] Z. Wang and J. Fang, Robust $H_{\infty}$ filter design with variance constraints and parabolic pole assignment, IEEE Signal Processing Letters, Vol. 13, No. 3, 2006, pp. 137-140.

[11] A. Hotz and R. E. Skelton, A covariance control theory, International Journal of Control, Vol. 46, No. 1, 1987, pp. 13-32.

[12] Y. Hung and Y. Fuwen, Robust $\mathcal{H}_{\infty}$ filtering with error variance constraints for discrete time-varying systems with uncertainty, Automatica, Vol. 39, 2003, pp. 1185-1194.

[13] Z. Wang, F. Yang and X. Liu, Robust filtering for systems with stochastic non-linearities and deterministic uncertainties, Proc. IMechE Part I: J. Systems and Control Engineering, Vol. 220, 2006, pp. 171-182.

[14] D. Ding, Z. Wang, B. Shen and H. Dong, Envelope-constrained $\mathcal{H}_{\infty}$ filtering with fading measurements and randomly occurring nonlinearities: the finite horizon case, Automatica, Vol. 55, May 2015, pp. 37-45.

[15] D. Ding, Z. Wang, J. Lam and B. Shen, Finite-Horizon $\mathcal{H}_{\infty}$ control for discrete time-varying systems with randomly occurring nonlinearities and fading measurements, IEEE Transactions on Automatic Control, (ISSN 0018-9286) In press (DOI: 10.1109/TAC.2014.2380671)

[16] R. Kalman and R. Bucy, A new approach to linear filtering and prediction problems, ASME Journal of Basic Engineering, Vol. 82, 1960, pp. 34-45.

[17] D. Bernstein and W. Haddad. Steady-state Kalman filtering with an $\mathcal{H}_{\infty}$ error bound. Systems $E^{3}$ Control Letters, Vol. 12, No. 1, 1989, pp. 9-16.

[18] H. Rotstein, M. Sznaier and M. Idan. Mixed $\mathcal{H}_{2} / \mathcal{H}_{\infty}$ filtering theory and an aerospace application. International Journal of Robust and Nonlinear Control, vol. 6, 1996, pp. 347-366.

[19] J. Hu, Z. Wang, H. Gao and L. K. Stergioulas, Extended Kalman filtering with stochastic nonlinearities and multiple missing measurements, Automatica, Vol. 48, No. 9, 2012, pp. 2007-2015.

[20] H. Gao, J. Lam and C. Wang, Induced $l_{2}$ and generalized $\mathcal{H}_{\infty}$ filtering for systems with repeated scalar nonlinearities, IEEE Transactions on Signal Processing, Vol. 53, No. 11, 2005, pp. 4215-4226.

[21] H. Gao, J. Lam and C. Wang, New approach to mixed $\mathcal{H}_{2} / \mathcal{H}_{\infty}$ filtering for polytopic discrete-time systems, IEEE Transactions

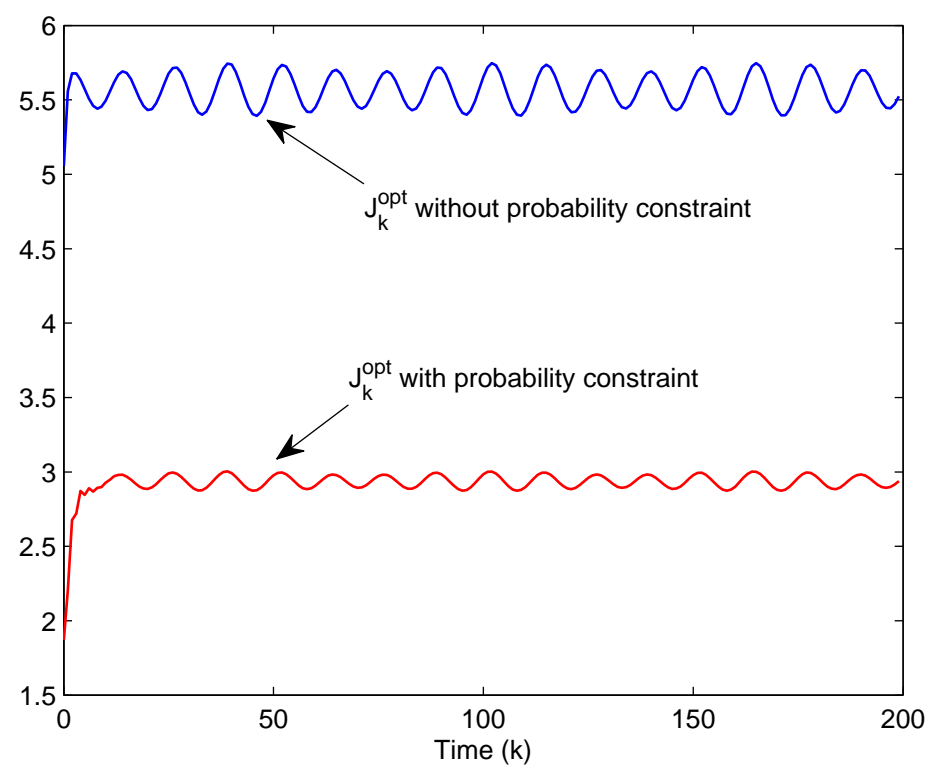

Fig. 3. Comparison of $J_{k}^{\text {opt }}$ in different cases 
on Signal Processing, Vol. 53, No. 8, 2005, pp. 3183-3192.

[22] H. Gao, J. Lam and C. Wang, Robust $\mathcal{H}_{\infty}$ filtering for discrete stochastic time-delay systems with nonlinear disturbances, Nonlinear Dynamics and System Theory, Vol. 4, No. 3, 2004, pp. 285-301.

[23] H. Gao, J. Lam and C. Wang, A delay-dependent approach to robust $\mathcal{H}_{\infty}$ filtering for uncertain discrete-time state-delayed systems, IEEE Transactions on Signal Processing, Vol. 52, No. 6, 2004, pp. 1631-1640.

[24] E. Gershon, U. Shaked and I. Yaesh, $\mathcal{H}_{\infty}$ control and filtering of discrete-time stochastic systems with multiplicative noise, Automatica, Vol. 37, 2001, pp. 409-417.

[25] F. Wang and V. Balakrishnan, Robust Kalman filters for linear time-varying systems with stochastic parametric uncertainties, IEEE Transactions on Signal Processing, Vol. 50, 2002, pp. 803-813.

[26] S. A. Kassam and H. V. Poor, Robust techniques for signal processing: a survey, Proceedings of the IEEE, Vol. 73, No. 3, 1985, pp. 433C481.

[27] C. V. Rao, J. B. Rawlings and D. Q. Mayne, Constrained state estimation for nonlinear discrete-time systems: stability and moving horizon approximations, IEEE Transactions on Automatic Control, Vol. 48, 2003, pp. 246-258.

[28] A. Alessandri, M. Baglietto and G. Battistelli, A minimax receding-horizon estimator for uncertain discrete-time linear systems, Proceedings of the American Control Conference, 2004, pp. 205-210.

[29] M. Rotea and C. Lana, State estimation with probability constraints, International Journal of Control, Vol. 81, No. 6, 2008, pp. 920-930.

[30] M. M. Kogan, Optimal estimation and filtration under unknown covariances of random factors, Automation and Remote Control, Vol. 75, No. 11, 2014, pp. 1964-1981.

[31] H. Dong, Z. Wang, J. Lam and H. Gao, Distributed filtering in sensor networks with randomly occurring saturations and successive packet dropouts, International Journal of Robust and Nonlinear Control, Vol. 24, No. 12, 2014, pp. 1743-1759.

[32] L. Ma, Y. Bo, Y. Zhou and Z. Guo, Error variance-constrained $H_{\infty}$ filtering for a class of nonlinear stochastic systems with degraded measurements: the finite horizon case, International Journal of Systems Science, Vol. 43, No. 12, 2012, pp. 2361-2372.

[33] D. Ding, Z. Wang, B. Shen and H. Dong, Envelope-constrained $H_{\infty}$ filtering with fading measurements and randomly occurring nonlinearities: the finite-horizon case, Automatica, Vol. 55, 2015, pp. 37-45.

[34] B. Shen, Z. Wang, H. Shu and G. Wei, $H_{\infty}$ filtering for uncertain time-varying systems with multiple randomly occurred nonlinearities and successive packet dropouts, International Journal of Robust and Nonlinear Control, Vol. 21, No. 14, 2011, pp. 1693-1709.

[35] Y. Luo, G. Wei, Y. Liu and X. Ding, Reliable $H_{\infty}$ state estimation for 2-D discrete systems with infinite distributed delays and incomplete observations, International Journal of General Systems, Vol. 44, No. 2, 2015, pp. $155-168$.

[36] Y. Yaz and E. Yaz, On LMI formulations of some problems arising in nonlinear stochastic system analysis, IEEE Transactions on Automatic Control, V0l.44, No.4, 1999, pp.813-816. 